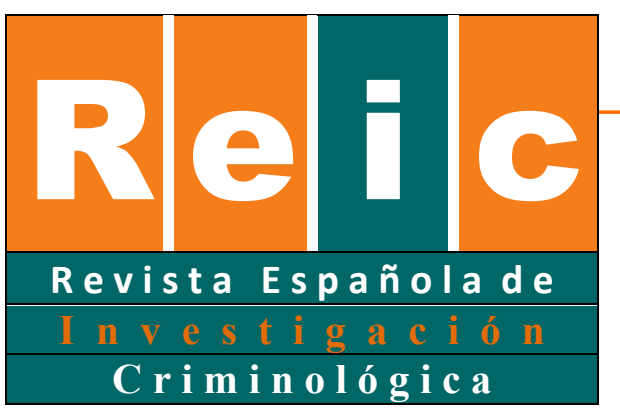

Cano \& Calvo

\title{
Evolución del miedo al delito y del punitivismo en la población universitaria española a partir de una muestra de estudiantes de Derecho
}

Evolution of fear of crime and punitivism in the Spanish university population from a sample of Law students

Miguel Ángel Cano Paños ${ }^{1}$ iD

Profesor Titular de Derecho Penal y Criminología. Universidad de Granada

María Ángeles Calvo Alba (D)

Investigadora predoctoral. Universidad de Granada

\section{RESUMEN}

Desde hace unos años la criminología española ha abordado a través de distintos estudios las actitudes punitivas de los ciudadanos. Sin embargo, son contados los trabajos en los que la muestra encuestada estaba formada por población universitaria. Por ello, el objetivo de este artículo es presentar los resultados de un estudio empírico de corte transversal repetido realizado con estudiantes universitarios matriculados en $1^{\text {er }}$ curso -130 alumnos- y en $4^{\circ}$ curso -102 alumnos- del Grado en Derecho en la Universidad de Granada, utilizando para ello una encuesta autoadministrada. En un estudio previo, publicado en el año 2019, los estudiantes de $1^{\mathrm{er}}$ curso encuestados percibían la situación de la delincuencia en España como amenazante y peligrosa, mostrando además unas actitudes punitivas de carácter severo. Tras confrontar esos resultados con los obtenidos con la muestra de $4^{\circ}$ curso, se puede observar cómo, en líneas generales, la percepción de la delincuencia, los niveles de miedo al delito y las actitudes punitivas disminuyen, en algunos casos de forma considerable, cuando se

\footnotetext{
${ }^{1}$ La correspondencia debe dirigirse a: Miguel Ángel Cano Paños. Email: macano@ugr.es
}

Revista Española de Investigación Criminológica

Artículo 5, Número 18 (2020)

DOI: https://doi.org/10.46381/reic.v18i0.377

www.criminologia.net

ISSN: 1696-9219 
compara la muestra de $1^{\mathrm{er}}$ curso con la de $4^{\mathrm{o}}$. Al mismo tiempo, las mujeres, tanto de $1^{\mathrm{er}}$ curso como de $4^{\circ}$, muestran, en general, unos niveles de miedo al delito y de punitivismo superiores a los existentes en la muestra de sexo masculino.

Palabras clave: Actitudes punitivas, miedo al delito, victimización, estudiantes universitarios, populismo punitivo

\section{ABSTRACT}

For some years, Spanish criminology has addressed through different studies the punitive attitudes of citizens. However, the works in which the surveyed sample consisted of university population are few. Therefore, the aim of this paper is to present the results of a repeated cross-sectional empirical study conducted with university students enrolled in the first year of the Degree in Law - 130 students - and in the fourth year - 102 students - both of the University of Granada, using a self-administered survey. In a previous study, published in 2019 by the authors, students enrolled in the first year of the Degree in Law perceived the situation of crime in Spain as threatening and dangerous, also showing punitive attitudes of a severe nature. After confronting these results with those obtained with the sample of students enrolled in the $4^{\text {th }}$ year, it can be observed how, in general terms, the perception of crime, the levels of fear of crime and punitive attitudes decrease, in some cases considerably. At the same time, women, both of the first year and 4th year of the Degree in Law, show, in general, levels of fear of crime and punitiveness higher than those existing in the male sample.

Keywords: punitive attitudes, fear of crime, victimization, university students, penal populism

\section{Introducción}

Ha sido fundamentalmente en la criminología desarrollada en los países anglosajones donde, desde la década de 1990, se han realizado investigaciones para analizar las actitudes de la ciudadanía frente a la delincuencia y, ligado a ello, para determinar la eficacia del sistema penal (Roberts \& Hough, 2005). Una de las razones que han dado lugar a un aumento significativo de estas investigaciones ha sido la consolidación del binomio «sistema penal represivo y demandas sociales de severidad» y, unido a ello, el mito existente -rechazado ampliamente por la criminología, tanto española (Varona Gómez, 2016), como internacional (entre otros Cullen, Cullen \& Wozniak, 1988)- según el cual la expansión del Derecho penal

Revista Española de Investigación Criminológica

Artículo 5, Número 18 (2020)

DOI: https://doi.org/10.46381/reic.v18i0.377

www.criminologia.net

ISSN: 1696-9219 
promovido por los órganos de decisión política tendría su origen en las demandas punitivas y de «mano dura» contra el delito provenientes de la ciudadanía.

En el caso de España, hasta épocas recientes no se ha contado con estudios criminológicos sobre las actitudes punitivas de los ciudadanos y su eventual relación con el miedo al delito (Aizpurúa, 2015; Aizpurúa González \& Fernández Molina, 2011; Fernández Molina \& Tarancón Gómez, 2010, Gómez Bellvís \& Falces Delgado, 2019; Varona Gómez, 2016). Al respecto conviene señalar que los orígenes de las investigaciones criminológicas destinadas a evaluar las actitudes punitivas de los ciudadanos españoles, así como su valoración del fenómeno delictivo, se encuentran en las encuestas de victimización llevadas a cabo en distintas partes del territorio nacional ya desde mediados de la década de 1990 (Díez Ripollés et al., 1996; García España, Pérez Jiménez \& Benítez Jiménez 2006; Luque Reina, 1999).

Ciertamente, el Estado español cuenta con toda una serie de fuentes de datos oficiales que aportan un conocimiento más o menos sólido sobre las actitudes punitivas reinantes en la ciudadanía y su valoración sobre el funcionamiento del sistema penal. Baste citar aquí los datos suministrados por los distintos Eurobarómetros ${ }^{2}$ o los estudios desarrollados por el Centro de Investigaciones Sociológicas (CIS), los cuales, ambos, debido en ocasiones a la utilización de preguntas genéricas dirigidas a los ciudadanos, así como al desconocimiento previo sobre el nivel de comprensión de esos mismos ciudadanos sobre la delincuencia o el funcionamiento de la justicia penal, deben sin embargo ser analizados y valorados con bastante cautela (Varona Gómez, 2009, pp. 11-12).

Ahora bien, un sector de la población que hasta ahora había sido desatendido en España a la hora de realizar encuestas sobre percepción de la delincuencia, miedo al delito y actitudes punitivas era el de los estudiantes de Derecho. Existe ciertamente un estudio pionero llevado a cabo con una muestra representativa de estudiantes universitarios españoles, en concreto de la Universidad de Girona (Varona Gómez, 2008), donde se

\footnotetext{
${ }^{2}$ Los Eurobarómetros son estudios sobre la opinión pública de los ciudadanos de la Unión Europea que la Comisión Europea lleva encargando desde el año 1973. Las encuestas que se llevan a cabo abarcan un amplio abanico de temas, entre los que se encuentran aspectos como la seguridad. Las mismas pueden consultarse en la siguiente dirección: http://www.europarl.europa.eu/at-your-service/es/be-heard/eurobarometer .
}

Revista Española de Investigación Criminológica

Artículo 5, Número 18 (2020)

DOI: https://doi.org/10.46381/reic.v18i0.377

www.criminologia.net

ISSN: 1696-9219 
analizaba su actitud frente a la prevención y castigo de la delincuencia, si bien el mismo no se limitaba a sondear la opinión de los estudiantes de ciencias jurídicas, teniendo por el contrario un carácter más general. Posteriormente, ha de destacarse también el estudio empírico llevado a cabo por García España, el cual estaba enfocado a analizar las actitudes punitivas de los estudiantes de $1^{\text {er }}$ curso del Grado de Criminología, y a un grupo de control formado por estudiantes de $1^{\circ}$ de Derecho, ambos matriculados en la Universidad de Málaga (García España, 2013). A nivel internacional conviene destacar el estudio realizado por Kohm et al. (2012) con estudiantes de cuatro centros universitarios, tres situados en EE. UU. y uno en Canadá, estando la muestra circunscrita a estudiantes de distintas áreas. El objetivo del estudio era testar el impacto que tienen los medios de comunicación en el miedo al delito mostrado por los estudiantes encuestados (Kohm, Waid-Lindberg, Weinrath, O'Connor \& Dobbs, 2012).

Desde el ámbito de la victimización, existen también a nivel nacional e internacional una serie de estudios que han abordado las experiencias de victimización en el seno del estudiantado universitario, tanto desde eventuales diferencias de género en función de la tipología delictiva en concreto, como en la relación de esa victimización con el miedo al delito y las actitudes punitivas (Barberet, Fisher \& Taylor, 2004; Chan-Gamboa, et al., 2017; Igareda \& Bodelón, 2014; Villacampa \& Pujols, 2017).

Lo descrito en los párrafos anteriores es desde luego sorprendente si se tiene en cuenta que el colectivo formado por estudiantes de Derecho, tras su formación jurídica, resulta determinante en la aplicación del Derecho, ya sea como jueces, fiscales o incluso como funcionarios de prisiones, pudiendo incluso influir decisivamente en la creación de la propia legislación penal. Si se parte del hecho de que los jóvenes que comienzan sus estudios de Derecho se encuentran precisamente, debido a su edad y a su estatus de estudiantes, en una fase de gran influenciabilidad -la cual, eventualmente, puede marcar su posterior actividad profesional-, en ese caso adquiere gran importancia conocer sus actitudes en torno a aspectos tales como la delincuencia, las penas y su eficacia.

Dejando de lado esta perspectiva de carácter especial, no puede tampoco ignorarse el hecho de que los estudiantes de Derecho son también parte de la sociedad, pudiendo, por

Revista Española de Investigación Criminológica

Artículo 5, Número 18 (2020)

DOI: https://doi.org/10.46381/reic.v18i0.377

www.criminologia.net

ISSN: 1696-9219 
tanto, pars pro toto, facilitar información relevante sobre las percepciones en torno a la delincuencia y los cambios en la mentalidad punitiva reinante en aquélla. En este sentido, y teniendo en cuenta la evolución política, jurídica y social acaecida en España en los últimos años, se plantea la pregunta de hasta qué punto se han producido cambios importantes en la mentalidad punitiva en el seno de la población española.

A la vista de estas circunstancias descritas, en los últimos años se ha llevado a cabo en la Universidad de Granada una investigación empírica de corte transversal repetido con estudiantes de Derecho matriculados en el primer curso. En concreto, se eligió una muestra en los cursos 2014/2015, 2015/2016, 2016/2017 y 2017/2018. A través de la administración de un cuestionario anónimo, el objetivo de la investigación era: (1) recabar la opinión de los estudiantes de $1^{\mathrm{er}}$ curso sobre cuestiones relacionadas con la situación de la delincuencia en España, su eventual victimización, así como sus actitudes punitivas; (2) analizar si los patrones de respuesta a las preguntas planteadas se mantenían invariables a lo largo de los años, o si más bien sufrían modificaciones de mayor o menor calado.

Los resultados de dicha investigación vieron la luz en forma de artículo científico en el año 2019 (Cano Paños \& Calvo Alba, 2019), si bien, en las conclusiones de la mencionada publicación, se suscitaba ya la siguiente pregunta: ¿Las tendencias de respuesta perceptibles en el alumnado de primer curso del Grado en Derecho con respecto a las variables relativas al miedo al delito, victimización y punitivismo persisten cuando dichas encuestas se llevan a cabo, no al comienzo de los estudios universitarios, sino poco antes de concluirlos? Es decir, ¿los patrones de respuesta se mantienen más o menos invariables entre los alumnos de cuarto curso? Pues bien, para responder a esta interesante cuestión, los autores de este estudio comenzaron durante el curso 2017/2018 a utilizar el mismo cuestionario, pero con estudiantes de cuarto curso matriculados también en el Grado en Derecho de la Universidad de Granada.

Por consiguiente, el objetivo fundamental del presente trabajo, continuación del anterior publicado en el año 2019, es presentar los resultados obtenidos con una muestra de estudiantes del Grado en Derecho que se encontraban matriculados en el último curso, y a los cuales se les administró el mismo cuestionario contestado por los alumnos de $1^{\mathrm{er}}$ curso. De este modo, el objetivo fundamental del estudio empírico es confrontar los patrones de

\section{Revista Española de Investigación Criminológica}

Artículo 5, Número 18 (2020)

DOI: https://doi.org/10.46381/reic.v18i0.377

www.criminologia.net

ISSN: 1696-9219 
respuesta otorgados por los estudiantes que se encuentran a las puertas de la graduación (curso 2017/2018), con aquellos manifestados por los alumnos que comenzaban sus estudios (curso 2014/2015). En relación con ello, la hipótesis fundamental que se plantea en el presente trabajo es que tanto el miedo al delito en su vertiente cognitiva -es decir, la percepción del riesgo de convertirse en víctima- como las actitudes punitivas del estudiantado disminuyen de forma apreciable con la progresiva formación académica. Al respecto hay que decir que la hipótesis que señala que a mayor nivel educativo hay menos miedo al delito, así como menos actitud punitiva, ha sido contrastada tanto a nivel nacional (Serrano Maíllo, 2016; Varona Gómez, 2013 y 2016) como internacional (Chen \& Einat, 2015). En concreto, en este último estudio, el cual tenía como objetivo testar las actitudes punitivas de una muestra de oficiales de policía y funcionarios de prisiones matriculados en estudios universitarios relacionados con la criminología o la justicia criminal, se pudo constatar que los funcionarios de prisiones (no así los agentes de policía) que se encontraban en el último año de carrera mostraban unas actitudes punitivas más laxas que al principio de sus estudios. Con todo, la investigación criminológica, nacional e internacional, si bien ha trabajado con el binomio nivel educativo en general y actitudes punitivas, lo ha hecho menos con respecto al nivel educativo de los estudiantes de un Grado relacionado tan directamente con la justicia penal como es el Grado en Derecho

Por último, un objetivo adicional en el presente trabajo es comprobar si la percepción de la delincuencia, el miedo al delito y las actitudes punitivas del estudiantado del sexo femenino presentan, en relación con los sujetos de sexo masculino, las mismas diferencias en $4^{\circ}$ curso que las que se producen en $1^{\circ}$. Al respecto hay que decir que la investigación empírica tanto nacional (Serrano Maíllo, 2016), como comparada (Applegate et al., 2002, pp. 96-97; Kury et al., 2002, p. 100) señala que, en líneas generales, las mujeres son menos punitivas que los hombres, si bien existen excepciones. 


\section{Metodología}

La base de datos del estudio que aquí se presenta consta de dos encuestas de corte transversal realizadas los años 2015 y 2018 a jóvenes de ambos $\operatorname{sexos}^{3}$ que, bien habían iniciado sus estudios de Derecho en la Universidad de Granada (estudiantes matriculados en el curso 2014/2015), o bien estaban a punto de concluirlos (curso 2017/2018), y cuyos resultados debían mostrar eventuales cambios en los dos cursos encuestados en lo relativo a aspectos tales como la percepción de la criminalidad, el miedo al delito o las actitudes punitivas.

Los estudiantes de $1^{\text {er }}$ curso encuestados, en el momento de pasar los cuestionarios, se encontraban matriculados en la asignatura «Derecho Penal I» (Parte General), y fue precisamente en esta asignatura cuando los mismos fueron contestados. Por su parte, los cuestionarios respondidos por el alumnado matriculado en $4^{\circ}$ curso se repartieron en distintas asignaturas optativas. En ambos casos, las encuestas se realizaron durante el mes de mayo del curso respectivo. De este modo, el alumnado de $1^{\circ}$ tenía ya una base de conocimientos sobre la ciencia jurídica en general y sobre el Derecho penal en particular, lo cual hacía que estuviesen familiarizados con la mayoría de las preguntas que conformaban la encuesta. Los cuestionarios se contestaron siempre en el aula donde se impartía la docencia, ya fuese antes de comenzar la clase, ya fuese al finalizar la misma ${ }^{4}$. En ambos casos, los cuestionarios fueron respondidos en papel de forma anónima e individual en presencia de uno de los autores del presente estudio, tratándose por tanto de encuestas autoadministradas. Tanto de forma oral (por parte del encuestador), como de forma escrita (en la primera hoja del cuestionario) se les indicó a los participantes en la encuesta que la misma no pretendía en absoluto evaluar los conocimientos de aquellos, sino que más bien el objetivo era conocer las percepciones personales de los encuestados en torno a distintas cuestiones; señalándoles, además, el carácter voluntario de la encuesta. Hay que decir que los estudiantes no recibieron incentivo

\footnotetext{
${ }^{3}$ En el presente trabajo, y con el fin de evitar la sobrecarga gráfica que supondría utilizar la constante terminación en «o/a» para una marcada existencia de ambos sexos, se ha optado por emplear el masculino genérico clásico, dejando claro que todas las menciones en tal género representan siempre a hombres y mujeres. ${ }^{4}$ En el estudio llevado a cabo por VARONA GÓMEZ en la Universidad de Girona, los cuestionarios fueron enviados vía e-mail a toda la muestra, por lo que en este caso no hubo un contacto directo entre el investigador y los encuestados.
}

Revista Española de Investigación Criminológica

Artículo 5, Número 18 (2020)

DOI: https://doi.org/10.46381/reic.v18i0.377

www.criminologia.net

ISSN: 1696-9219 
alguno por su participación en el estudio. Por lo demás, el tiempo de cumplimentación del cuestionario osciló entre los 10 y los 15 minutos.

El cuestionario utilizado en esta investigación es una traducción directa del alemán y adaptación del creado y desarrollado en su día en Alemania por Franz Streng, Profesor emérito de Derecho Penal y Criminología en la Friedrich-Alexander Universität ErlangenNürnberg. En su versión original, dicho cuestionario fue utilizado en un estudio aparecido en Alemania en el año 2014 titulado «La transformación en la percepción de la criminalidad y de la punitividad. Actitudes en torno a la criminalidad y la orientación profesional de jóvenes juristas $»^{5}$. En la versión española se añadieron una serie de preguntas que no figuraban en la versión original en alemán.

En lo que hace referencia al tamaño de la muestra en los dos cursos encuestados, la misma se desglosó de la siguiente manera: curso 2014/2015: 130 alumnos -37 hombres y 93 mujeres-; curso 2017/2018: 102 alumnos -34 hombres y 68 mujeres-. Teniendo en cuenta que en la Universidad de Granada existen un total de nueve grupos (de las letras A a I) matriculados en $1^{\text {er }}$ curso del Grado en Derecho, los cuestionarios se pasaron cada año en tres de esos grupos. Por su parte, para el alumnado matriculado en cursos superiores, se decidió pasar los cuestionarios, no en cursos específicos, sino en distintas asignaturas optativas, ya que en algunas de ellas la asistencia no superaba los 5-8 alumnos, mientras que en otras la cifra alcanzaba de largo los 40. El objetivo era lograr un tamaño de la muestra semejante a la del $1^{\text {er }}$ curso. Hay que decir que la tasa de respuesta fue del $100 \%$, y ello teniendo en cuenta que, antes de repartir los cuestionarios, se les indicó a los estudiantes el carácter voluntario del mismo. Además, no se excluyó a ningún participante ya que la totalidad de los cuestionarios fueron respondidos completamente.

Una vez recogidos los cuestionarios y creada la base de datos en SPSS, la primera fase del estudio - de naturaleza descriptiva- comenzó con la confección del perfil sociodemográfico del estudiantado matriculado en $1^{\text {er }}$ curso y en $4^{\circ}$ curso del Grado en Derecho. Para ello, se utilizaron las siguientes variables: (1) sexo; (2) edad; (3) ámbito

\footnotetext{
${ }^{5}$ Título original: «Kriminalitätswahrnehmung und Punitivität im Wandel. Kriminalitäts- und berufsbezogene Einstellungen junger Juristen», Ed. Kriminalistik, Heidelberg, 2014.
}

Revista Española de Investigación Criminológica

Artículo 5, Número 18 (2020)

DOI: https://doi.org/10.46381/reic.v18i0.377

www.criminologia.net

ISSN: 1696-9219 
geográfico de procedencia; (4) nivel socioeconómico de origen; (5) opinión sobre la función de la justicia; (6) expectativa laboral.

Para una mayor profundidad en el conocimiento sobre la composición muestral, el siguiente paso fue detallar, en cada uno de los dos cursos académicos encuestados, la percepción de los estudiantes sobre el estado de la delincuencia en España y las experiencias personales que habían tenido siendo víctimas de algún delito. Para ello, las variables seleccionadas con vistas a abordar el miedo al delito/victimización de los estudiantes fueron cuatro: (1) la valoración en general de la delincuencia en España; (2) la valoración sobre la percepción de verse expuesto a un ataque violento por parte de una tercera persona; (3) el haber sido víctima de una agresión física o sexual en los últimos tres años y (4) el haber sido víctima de un hurto/robo en los últimos tres años.

La fase descriptiva del estudio concluyó con la distribución porcentual de la actitud punitiva en los dos cursos académicos encuestados, empleándose para ello la opinión que los estudiantes tenían sobre la pena de prisión permanente revisable (PPR) y sobre la pena de muerte $(\mathrm{PM})^{6}$.

La segunda fase de la investigación fue más analítica, de corte bivariada. El objetivo principal era determinar la posible existencia de diferencias en las inclinaciones punitivas de los encuestados en función del sexo; y ello a partir también del curso académico en el que se respondió al cuestionario. Para ello se emplearon las variables sexo y actitud punitiva, diseccionada esta última entre la PPR y la PM, tomando también en consideración los mayores o menores niveles de miedo al delito mostrados por los estudiantes en función precisamente del sexo. El procedimiento seguido para cumplir con esta segunda fase comenzó con la segmentación de la muestra en hombres y mujeres a través de la selección de casos, a fin de poder comparar los resultados obtenidos por ambos sexos, tanto en $1^{\circ}$ como

\footnotetext{
${ }^{6} \mathrm{Y}$ ello teniendo en cuenta que en España existen ya estudios (Aizpurúa, 2015), los cuales han medido el punitivismo utilizando para ello escalas compuestas por indicadores múltiples, las cuales, lógicamente, miden el fenómeno de manera más comprehensiva. De hecho, el propio cuestionario que se administró al alumnado contenía otras preguntas dirigidas indirectamente a medir el punitivismo, si bien las mismas no se han utilizado para la elaboración del presente trabajo.
}

Revista Española de Investigación Criminológica

Artículo 5, Número 18 (2020)

DOI: https://doi.org/10.46381/reic.v18i0.377

www.criminologia.net

ISSN: 1696-9219 
en $4^{\circ}$ curso del Grado en Derecho, en las cuestiones relacionadas con las actitudes punitivas y la victimización.

De manera más concreta, el análisis bivariado se realizó mediante tablas de contingencia en las que se expusieron los resultados y diferencias encontradas por curso académico y sexo en torno a las variables de valoración de la PPR, de la PM, la valoración general de la delincuencia en España, el riesgo de verse expuesto a un ataque violento por parte de una tercera persona y el haber sido víctima de una agresión o de un robo/hurto - en ambos casos, en los últimos 3 años. En lo que respecta a las variables de miedo al delito y de haber sido víctima de alguno de los delitos mencionados, el análisis de las diferencias que se producen entre hombres y mujeres -teniendo para ello en cuenta el curso académico- se realizó mediante la selección de casos: en primer lugar, se seleccionaron a los hombres para conocer las diferencias entre los varones de $1^{\circ}$ con respecto a los de $4^{\circ}$. Dicho proceso fue replicado para el caso de las mujeres, facilitando con ello la comparación de los cambios entre cursos en función del sexo.

Para saber si las diferencias entre las variables cruzadas eran estadísticamente significativas, se empleó chi cuadrado de Pearson y V de Cramer para determinar el tamaño del efecto -pequeño: 0,06<0,17; medio: 0,17<0,29; grande: $\geq 0.29)$ (Cohen, 1988).

Teniendo en cuenta todo lo explicado, en los epígrafes siguientes se va a comparar la evolución de la percepción que tienen los estudiantes de Derecho de la Universidad de Granada sobre la situación de la delincuencia en España, su victimización (real o percibida), así como sus concepciones en torno al castigo penal. Para ello, se van a confrontar los datos obtenidos del alumnado de $4^{\circ}$ durante el curso 2017/2018 ( $\left.\mathrm{N}=102\right)$, con aquellos que se obtuvieron previamente en la muestra de alumnos matriculados en $1^{\circ}$ durante el curso 2014/2015 (N=130). De este modo se pueden extraer datos interesantes sobre la evolución de la percepción del estudiantado en torno a las cuestiones planteadas, y ello tanto al inicio como a la finalización de los estudios de Derecho. 


\section{Resultados}

\subsection{Análisis sociodemográfico y concepciones en torno al futuro profesional}

En $4^{\mathrm{o}}$ curso, el Grado en Derecho es una titulación mayormente cursada por mujeres (Tabla 1), aunque esta brecha, realmente notoria entre el estudiantado matriculado en $1^{\mathrm{er}}$ curso, se reduce ligeramente al llegar al último curso del grado, donde el porcentaje de hombres aumenta en 4,8 puntos porcentuales con respecto al $1^{\mathrm{er}}$ curso (de un 28,5\% a un 33,3\%). Por lo que hace referencia a la distribución etaria, y como era de prever, la edad media de los estudiantes ha aumentado, siendo tres años superior en $4^{\circ}$ curso que en $1^{\circ}$, aunque la desviación típica disminuye en un punto. En relación con el ámbito geográfico de procedencia no se producen cambios en la composición muestral entre $1^{\mathrm{o}} \mathrm{y} 4^{\mathrm{o}}$ : aumenta moderadamente la presencia de estudiantes procedentes de grandes y medianas ciudades, mientras que se reduce la de los que provienen de ciudades pequeñas y del ámbito rural.

La distribución del estudiantado en la clase social de origen muestra pequeñas variaciones en el aumento de estudiantes autodefinidos como clase media-alta (12,6 puntos más) en detrimento de los de clase media-baja y media (que pierden 5,4 y 7,9 puntos porcentuales, respectivamente).

La opinión que tienen los estudiantes de Derecho sobre la tarea que debe cumplir un jurista revela un gran cambio. En Primer curso, la mayor parte de los estudiantes consideraba que la principal tarea del jurista era impartir justicia (57,7\%); sin embargo, en $4^{\circ}$ curso, estos reconocen que la tarea principal del jurista debe ser evitar y regular conflictos sociales (47,0\%). Finalmente, sobre el ámbito profesional en el que les gustaría desarrollar su actividad una vez obtenida la titulación, no se encuentran grandes diferencias en $4^{\circ}$ curso con respecto al alumnado de $1^{\circ}$, siendo eso sí el funcionariado público el que más adeptos pierde (11,3 puntos porcentuales, si bien continúa como la primera opción escogida por los estudiantes), mientras que la categoría de «otros» es la que más crece (9,6 puntos más con respecto al curso 2014/2015). 


\section{Tabla 1.}

Perfil del estudiantado por curso académico (1 ${ }^{\text {er }}$ curso: 2014/2015; $4^{\circ}$ curso: 2017/2018)

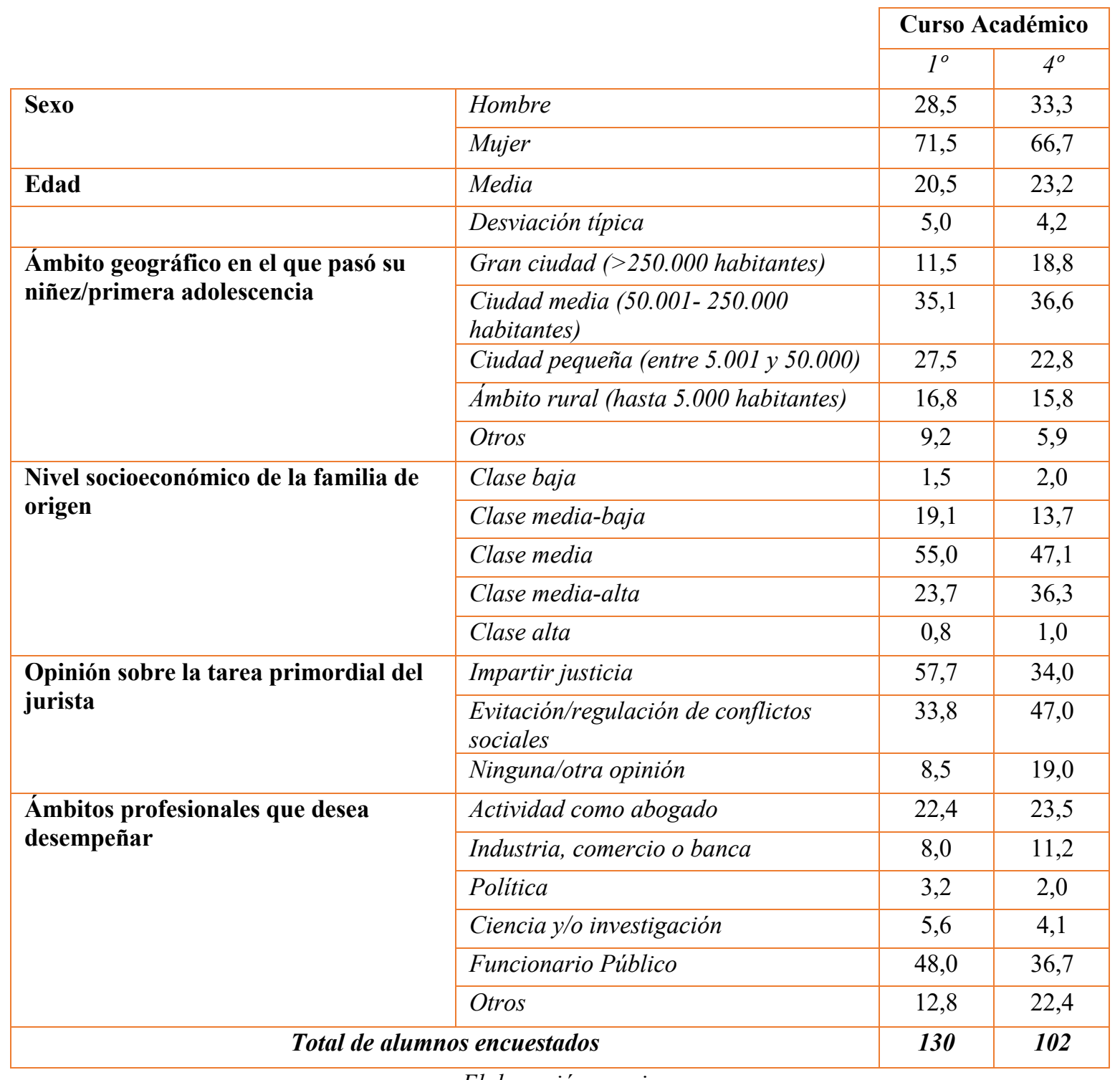

Elaboración propia

\subsection{Percepción de la delincuencia en España y victimización}

La evolución de las respuestas otorgadas por los alumnos encuestados en relación a las variables que miden la victimización (Tabla 2), muestra una variación importante al 
comparar los resultados obtenidos, según fuera el cuestionario cumplimentado en $1^{\mathrm{er}}$ curso o en $4^{\circ}$ curso del Grado en Derecho.

Con respecto a las variables relacionadas con la victimización percibida (valoración general de la delincuencia en España y valoración sobre el grado de exposición a un ataque violento por parte de una tercera persona), los resultados muestran que se produce una disminución porcentual de aquellos cuya percepción del entorno es más victimista. Así, la opción de respuesta que considera la situación de la delincuencia en España como «amenazante y peligrosa», disminuye en 19,1 puntos porcentuales entre los cursos $1^{\circ}$ y $4^{\circ}(\mathrm{de}$ un $53,8 \%$ a un $34,7 \%$ ), mientras que el «riesgo alto» de verse expuesto a un ataque violento por parte de una tercera persona lo hace en 11,5 puntos (de un 61,5\% a un 50,0\%). Paralelamente, se incrementa de forma notable el número de estudiantes cuya percepción se sitúa precisamente en el polo opuesto. Así, la opción de respuesta «como no peligrosa ni amenazante» en relación con la situación de la delincuencia en España se incrementa nada menos que en 34,9 puntos porcentuales (de un 19,2\% a un 54,1\%), mientras que la opción de respuesta «riesgo bajo» con respecto al grado de exposición a un ataque violento por parte de una tercera persona aumenta en 14,5 puntos (de un 28,5\% a un 43,0\%). Conviene también destacar cómo el porcentaje de indecisos («sin opinión») en relación con la situación de la delincuencia en España baja considerablemente entre $1^{\text {er }}$ y $4^{\circ}$ curso, pasando de un $26,9 \%$ a un $11,2 \%$.

Por otro lado, la victimización experimentada de forma directa por el estudiantado revela una tendencia similar a la percibida. Efectivamente, en lo que atañe al haber sido víctima de una agresión física o sexual en los últimos tres años se produce un ligero incremento en aquellos estudiantes de $4^{\circ}$ curso que sí han sido víctimas, tanto de los que no han sufrido daños personales (3,7 puntos porcentuales más) como de los que sí los han sufrido (1,1 puntos más). Ahora bien, en el caso del robo y el hurto para el mismo periodo se produce una situación diferente: si bien es cierto que aumenta en 3,8 puntos el porcentaje de estudiantes que no han sido víctimas de estas tipologías delictivas (de un 68,7\% a un 72,5\%), se puede observar cómo en cambio se incrementa ligeramente (en 0,2 puntos porcentuales) el número de los que han sido víctimas más de una vez (de un 7,6\% a un 7,8\%).

Revista Española de Investigación Criminológica

Artículo 5, Número 18 (2020)

DOI: https://doi.org/10.46381/reic.v18i0.377

www.criminologia.net

ISSN: 1696-9219 
Los resultados de chi cuadrado detallan que las diferencias estadísticamente significativas se producen sólo entre la valoración de la delincuencia en España y el curso académico en el que se está matriculado, siendo grande el tamaño del efecto (chi cuadrado=31,157; n.s. $=0,000 ; \mathrm{V}$ de Cramer $=0,370>0,29)$, es decir, que la percepción que los estudiantes tienen sobre esta cuestión varía de forma significativa si se compara la muestra de $1^{\text {er }}$ curso con la de $4^{\text {o }}$.

\section{Tabla 2.}

Victimización por curso académico

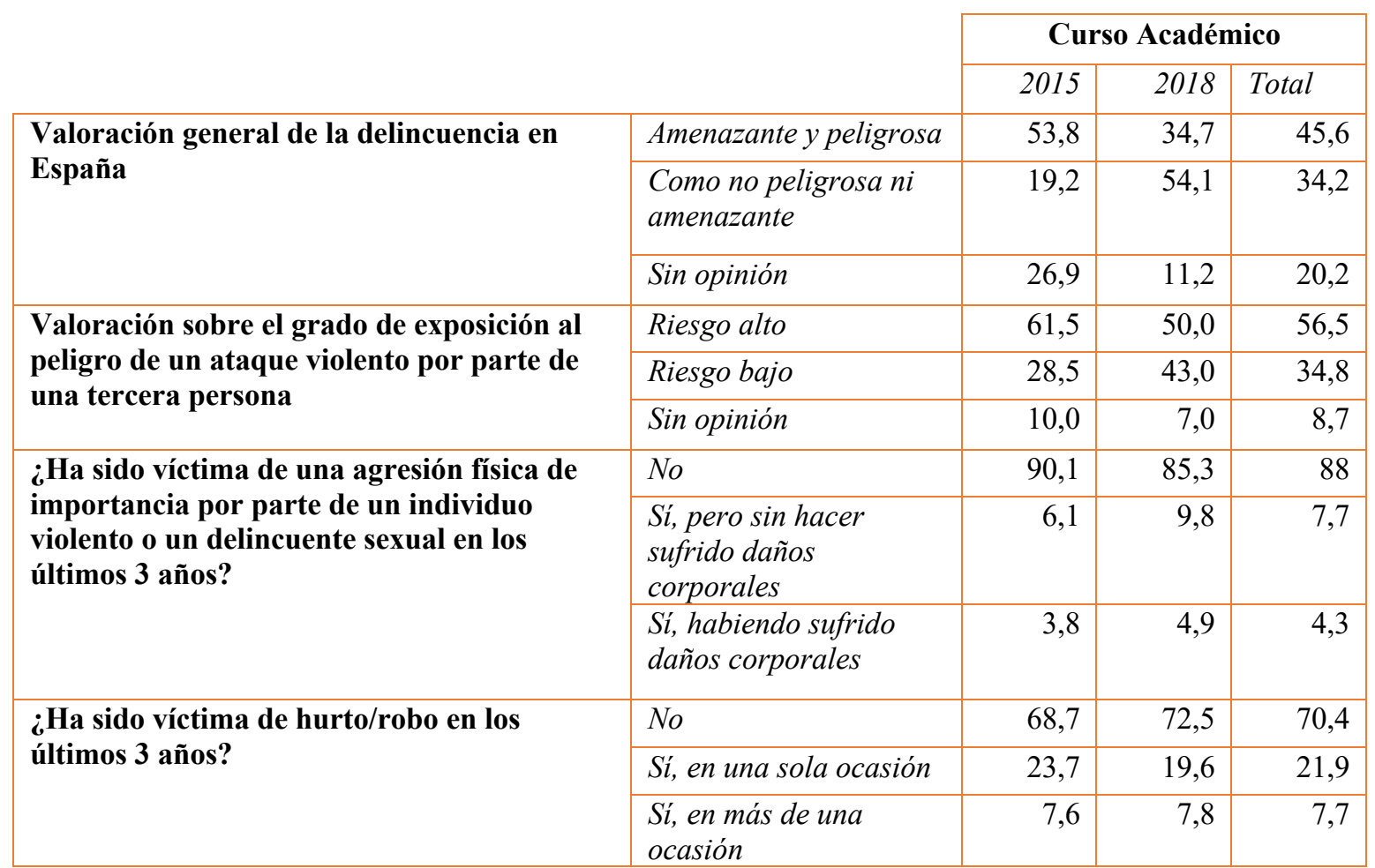

Elaboración propia

\subsection{Actitudes punitivas}

Por su parte, el punitivismo del estudiantado de $4^{\circ}$ de Derecho es también medido a través de la opinión que manifiestan tanto sobre la pena de prisión permanente revisable (PPR), como sobre la pena de muerte (PM) (Tabla 3). 
Lo primero que hay que señalar es que las actitudes más punitivas -es decir, aquellas que reclaman la existencia de una pena incluso más severa que la $\mathrm{PPR}^{7}$, o bien que claman por la regulación de la PM en el Código Penal- muestran una cierta disminución. Así, el número de aquellos estudiantes que consideran que debería haber una pena aún más grave que la PPR se reduce ligeramente en la muestra correspondiente a $4^{\circ}$ curso (pasando de un $24,1 \%$ en $1^{\text {er }}$ curso a un $21,1 \%$ en $4^{\circ}$ o, lo que es lo mismo, una disminución en 3 puntos porcentuales), mientras que los que están de acuerdo con la introducción de la PM en el Código Penal presentan una caída más acusada (13,3 puntos porcentuales, pasando de un $39,8 \%$ a un $26,5 \%$ ).

Esta disminución producida en las actitudes más punitivas de los estudiantes de Derecho entre $1^{\mathrm{er}}$ y $4^{\mathrm{o}}$ curso revierte sobre las menos punitivas. De este modo, el número de estudiantes de $4^{\circ}$ que piden la derogación de la PPR aumenta en 5,1 puntos porcentuales con respecto a los estudiantes de $1^{\circ}$ (pasando de un 10,7\% a un 15,8\%). De forma más acusada, los que se posicionan en contra de la PM aumentan nada menos que en 13,3 puntos (de un $60,2 \%$ en $1^{\text {er }}$ curso a un $73,5 \%$ en $4^{\circ}$ Curso).

Con todo, y en relación con el controvertido instituto jurídico de la PPR, los resultados obtenidos entre el estudiantado de $4^{\mathrm{o}}$ curso indican que, al igual que sucedía con $1^{\mathrm{er}}$ curso, son todavía mayoritarios los que consideran que esta pena debería ser aplicada de forma obligatoria para los delitos de especial gravedad (un $65,2 \%$ en $1^{\mathrm{er}}$ curso y un $63,2 \%$ en $4^{\circ}$ curso).

En esta ocasión, chi cuadrado muestra que existe asociación entre el grado de acuerdo que se tenga sobre la PM y el curso académico en el que se cumplimentó la encuesta, siendo el tamaño del efecto pequeño (chi cuadrado=4,233; n.s=0,040; V de Cramer $=0,140<0,29$ ), confirmando que la actitud punitiva que se refleja a través de la opinión que se tenga de la PM se ve influida por el curso en el que se encuentren matriculados los estudiantes de Derecho.

\footnotetext{
${ }^{7}$ En este caso, las opciones posibles serían únicamente la pena de prisión permanente sin posibilidad de revisión $o$ bien la pena de muerte.

Revista Española de Investigación Criminológica

Artículo 5, Número 18 (2020)

DOI: https://doi.org/10.46381/reic.v18i0.377

www.criminologia.net

ISSN: 1696-9219
} 


\section{Tabla 3.}

Punitivismo por curso académico

\begin{tabular}{|c|c|c|c|c|}
\hline & & \multicolumn{3}{|c|}{ Curso Académico } \\
\hline & & 2015 & 2018 & Total \\
\hline \multirow{3}{*}{$\begin{array}{l}\text { Opinión con } \\
\text { respecto a la PPR }\end{array}$} & Debería derogarse del Código Penal & 10,7 & 15,8 & 13,0 \\
\hline & $\begin{array}{l}\text { Debería aplicarse obligatoriamente en relación con } \\
\text { delitos de especial gravedad }\end{array}$ & 65,2 & 63,2 & 64,3 \\
\hline & Debería existir otra pena aún más grave & 24,1 & 21,1 & 22,7 \\
\hline \multirow{2}{*}{$\begin{array}{l}\text { Grado de acuerdo } \\
\text { sobre la } \\
\text { introducción de la } \\
\text { PM para ciertos } \\
\text { delitos en el } \\
\text { Código Penal }\end{array}$} & $S i$ & 39,8 & 26,5 & 33,8 \\
\hline & No & 60,2 & 73,5 & 66,2 \\
\hline
\end{tabular}

Elaboración propia

\subsection{Actitud punitiva y miedo al delito en función del sexo}

Con respecto a los estudiantes de Derecho matriculados en $4^{\circ}$ curso en la Universidad de Granada, las actitudes punitivas revelan diferencias entre los sexos, confirmando así una mayor presencia de punitivismo entre las mujeres que en el caso de los hombres (Tabla 4). Así, en relación con la PPR, el grueso de los encuestados se ubica en la categoría «neutra» ${ }^{8}$ (aplicación obligatoria para los delitos de especial gravedad), indistintamente de que se trate de hombres o de mujeres. Ahora bien, las diferencias entre $1^{\circ}$ y $4^{\circ}$ muestran la confirmación de una tendencia: si bien disminuyen ligeramente los porcentajes de hombres y mujeres que están a favor de aplicar penas más duras que la PPR, en el caso de las mujeres esta opción de respuesta sigue acumulando más del $20 \%$ de las encuestadas $\left(25,9 \%\right.$ en $4^{\circ}$ y $24,2 \%$ en $\left.1^{\circ}\right)$, llegando apenas al 15\% en el caso de los hombres matriculados en $4^{\circ}$ curso. Además, es en el grupo de hombres en el que se produce un mayor incremento de actitudes no punitivas si se compara la muestra de $1^{\mathrm{er}}$ curso con la de $4^{\mathrm{o}}$, si bien tales diferencias no son excesivamente

\footnotetext{
${ }^{8}$ El calificativo «neutra» se utiliza aquí exclusivamente por razones metodológicas, para con ello diferenciar las distintas opciones de respuesta que tenía el alumnado. Es evidente que abogar por el mantenimiento de la PPR, tal y como la misma se encuentra actualmente regulada en el Texto Punitivo, supone una manifestación de punitivismo.
}

Revista Española de Investigación Criminológica

Artículo 5, Número 18 (2020)

DOI: https://doi.org/10.46381/reic.v18i0.377

www.criminologia.net

ISSN: 1696-9219 
reseñables. Así, mientras que un $15,2 \%$ de los estudiantes de sexo masculino matriculados en $4^{\circ}$ se muestran contrarios a aplicar la PPR, dicho porcentaje sube a un $16,1 \%$ en el caso de sus compañeras de sexo femenino.

Con todo, es en la valoración de la PM donde se encuentra el mayor cambio entre $1^{\circ}$ y $4^{\circ}$ en función del sexo. Así, las mujeres matriculadas en $1^{\text {er }}$ curso muestran un mayor punitivismo que los hombres, al estar un 46,4\% de ellas a favor de regular la PM en el Código Penal, frente al $24,2 \%$ de los hombres. Sin embargo, en $4^{\circ}$ curso de carrera se reduce drásticamente el porcentaje de mujeres que se posicionan en esta categoría, disminuyendo en 19,8 puntos porcentuales para alcanzar un 26,6\%, mientras que paralelamente crece también en 19,8 puntos el grupo de las que se oponen a la introducción de la PM en el Código Penal (de un $53,6 \%$ en $1^{\text {er }}$ curso a un $73,4 \%$ en $4^{\circ}$ ). Cerca de romper la tendencia marcada por los resultados sobre la valoración de la PPR en función del sexo, en $4^{\circ}$ curso se igualan los hombres $(26,5 \%)$ y mujeres $(26,6 \%)$ que se muestran partidarios de regular la pena capital en el Texto Punitivo.

En esta ocasión, chi cuadrado determina que las diferencias que se producen en la valoración en el grupo de las mujeres en relación a la PM son estadísticamente significativas, aun siendo moderado el tamaño del efecto (chi cuadrado=6,095; n.s. $=0,014$; V de Cramer $=0,203<0,29)$, confirmando así la existencia de asociación en estos dos casos. En el caso de las diferencias halladas en torno a la valoración de la pena de PPR, las pruebas de chi cuadrado determinaron que no eran estadísticamente significativas. 


\section{Tabla 4.}

Punitivismo por sexo y curso académico

\begin{tabular}{|c|c|c|c|c|c|c|c|}
\hline & & \multicolumn{6}{|c|}{ Sexo } \\
\hline & & \multicolumn{3}{|c|}{ Hombre } & \multicolumn{3}{|c|}{ Mujer } \\
\hline & & \multicolumn{6}{|c|}{ Curso Académico } \\
\hline & & $1^{o}$ & $4^{\circ}$ & Total & $1^{o}$ & $4^{o}$ & Total \\
\hline \multirow{3}{*}{$\begin{array}{l}\text { Opinión con respecto a la } \\
\text { pena de prisión permanente } \\
\text { revisable }\end{array}$} & $\begin{array}{l}\text { Debería derogarse del } \\
\text { Código Penal }\end{array}$ & 10,0 & 15,2 & 12,7 & 11,1 & 16,1 & 13,3 \\
\hline & $\begin{array}{l}\text { Debería aplicarse } \\
\text { obligatoriamente en relación } \\
\text { con delitos de especial } \\
\text { gravedad }\end{array}$ & 70,0 & 69,7 & 69,8 & 63,0 & 59,7 & 61,5 \\
\hline & $\begin{array}{l}\text { Debería existir otra pena } \\
\text { aún más grave }\end{array}$ & 20,0 & 15,2 & 17,5 & 25,9 & 24,2 & 25,2 \\
\hline \multirow{2}{*}{$\begin{array}{l}\text { Grado de acuerdo sobre la } \\
\text { introducción de la pena de } \\
\text { muerte para ciertos delitos } \\
\text { en el Código Penal }\end{array}$} & $S i$ & 24,2 & 26,5 & 25,4 & 46,4 & 26,6 & 37,8 \\
\hline & No & 75,8 & 73,5 & 74,6 & 53,6 & 73,4 & 62,2 \\
\hline \multicolumn{2}{|c|}{ Tamaño muestral } & 37 & 34 & 71 & 93 & 68 & 161 \\
\hline
\end{tabular}

Elaboración propia

Por último, la evolución de la victimización percibida revela también diferencias entre ambos sexos (Tabla 5). Efectivamente, en la valoración que los estudiantes de sexo masculino y femenino de $1^{\mathrm{er}}$ curso hacían sobre el estado de la delincuencia en España, ambos grupos la consideraban mayoritariamente «como amenazante y peligrosa». Sin embargo, al llegar a $4^{\circ}$ se constata un cambio con respecto a esta percepción, creciendo sustancialmente el grupo de quienes no la consideran como amenazante ni peligrosa. Sin embargo, entre las mujeres sigue habiendo una leve mayoría que presenta una percepción victimista del entorno medido a través de esta concreta pregunta (un $39,1 \%$ de mujeres matriculadas en $4^{\circ}$ curso frente a un $26,5 \%$ de hombres).

Sobre la cuestión relativa al grado de exposición a ser víctimas de un ataque violento por parte de una tercera persona, las estudiantes de sexo femenino vuelven a mostrar una actitud más victimista que los hombres, al confesar que perciben un riesgo alto de que se produzca esta situación, y ello tanto en $1^{\circ}(75,0 \%)$ como en $4^{\circ}$ curso $(62,1 \%)$. Esto supone ciertamente una tendencia contraria a los hombres, los cuales tanto en $1^{\circ}(59,5 \%)$ como en $4^{\circ}$

Revista Española de Investigación Criminológica

Artículo 5, Número 18 (2020)

DOI: https://doi.org/10.46381/reic.v18i0.377

www.criminologia.net

ISSN: 1696-9219 
$(67,6 \%)$ afirman percibir un riesgo bajo. Por otro lado, también se observa cómo al analizar los cuestionarios en $4^{\circ}$ aumenta en 14,0 puntos porcentuales el número de mujeres que consideran que existe un riesgo bajo $\left(30,3 \%\right.$ en $4^{\circ}$ frente a un $16,3 \%$ en $\left.1^{\circ}\right)$.

Con respecto a la evolución de la diferencia entre sexos a la hora de indicar si se ha sido víctima de una agresión física o sexual en los últimos tres años, se observa cómo ésta se intensifica si se comparan los datos de $1^{\text {er }}$ curso con los de $4^{\mathrm{o}}$; y, aunque en ambos casos aumenta el número de víctimas, es en el grupo de las mujeres donde se aprecia esta tendencia con más intensidad.

Por su parte, el número de hombres que declaran no haber sido víctimas de un delito de robo/hurto en los últimos tres años se reduce en 4,9 puntos porcentuales (pasando de un $78,4 \%$ en $1^{\text {er }}$ curso a un $73,5 \%$ en $4^{\circ}$ ), mientras que el de mujeres aumenta en 6,5 puntos (de un $65,6 \%$ que en $1{ }^{\text {er }}$ Curso declaraban no haber sido víctimas de dichas tipologías delictivas a un $72,1 \%$ que lo hacen en $4^{\circ}$ curso). A destacar también es el hecho de que son las mujeres las que han sufrido más de un robo o hurto en los últimos tres años.

Chi cuadrado determina que las únicas diferencias estadísticamente significativas se encuentran en los cambios producidos entre $1^{\mathrm{er}} \mathrm{y} 4^{\mathrm{o}}$ curso del Grado en Derecho, en función del sexo, sobre las variables seleccionadas en torno a la valoración general de la delincuencia en España en el caso de los hombres (chi cuadrado=10,299; V de Cramer=0,381>0,29) y en el caso de las mujeres (chi cuadrado=21,239; n.s. $=0.000 ; \mathrm{V}$ de Cramer=0,369>0,29), ambos con un buen tamaño del efecto como indica $\mathrm{V}$ de Cramer. 


\section{Tabla 5.}

Victimización por sexo y curso académico

\begin{tabular}{|c|c|c|c|c|c|c|c|}
\hline & & \multicolumn{6}{|c|}{ Sexo } \\
\hline & & \multicolumn{3}{|c|}{ Hombre } & \multicolumn{3}{|c|}{ Mujer } \\
\hline & & \multicolumn{6}{|c|}{ Curso Académico } \\
\hline & & $1^{o}$ & $4^{o}$ & Total & $1^{\circ}$ & $4^{o}$ & Total \\
\hline \multirow{3}{*}{$\begin{array}{l}\text { Valoración general de } \\
\text { la delincuencia en } \\
\text { España }\end{array}$} & Amenazante y peligrosa & 54,1 & 26,5 & 40,8 & 54,3 & 39,1 & 48,1 \\
\hline & $\begin{array}{l}\text { Como no peligrosa } \mathrm{ni} \\
\text { amenazante }\end{array}$ & 29,7 & 67,6 & 47,9 & 14,1 & 46,9 & 27,6 \\
\hline & Sin opinión & 16,2 & 5,9 & 11,3 & 31,5 & 14,1 & 24,4 \\
\hline \multirow{3}{*}{$\begin{array}{l}\text { Valoración sobre el } \\
\text { grado de exposición al } \\
\text { peligro de un ataque } \\
\text { violento por parte de } \\
\text { una tercera persona }\end{array}$} & Riesgo alto & 27 & 26,5 & 26,8 & 75 & 62,1 & 69,6 \\
\hline & Riesgo bajo & 59,5 & 67,6 & 63,4 & 16,3 & 30,3 & 22,2 \\
\hline & Sin opinión & 13,5 & 5,9 & 9,9 & 8,7 & 7,6 & 8,2 \\
\hline \multirow{3}{*}{$\begin{array}{l}\text { ¿Ha sido víctima de } \\
\text { una agresión física de } \\
\text { importancia por parte } \\
\text { de un individuo } \\
\text { violento o un } \\
\text { delincuente sexual en } \\
\text { los últimos } 3 \text { años? }\end{array}$} & No & 91,9 & 88,2 & 90,1 & 89,2 & 83,8 & 87 \\
\hline & $\begin{array}{l}\text { Si, pero sin hacer sufrido } \\
\text { daños corporales }\end{array}$ & 2,7 & 5,9 & 4,2 & 7,5 & 11,8 & 9,3 \\
\hline & $\begin{array}{l}\text { Si, habiendo sufrido daños } \\
\text { corporales }\end{array}$ & 5,4 & 5,9 & 5,6 & 3,2 & 4,4 & 3,7 \\
\hline \multirow{3}{*}{$\begin{array}{l}\text { ¿Ha sido víctima de } \\
\text { hurto/robo en los } \\
\text { últimos } 3 \text { años? }\end{array}$} & No & 78,4 & 73,5 & 76,1 & 65,6 & 72,1 & 68,3 \\
\hline & Sí, en una sola ocasión & 18,9 & 23,5 & 21,1 & 25,8 & 17,6 & 22,4 \\
\hline & Sí, en más de una ocasión & 2,7 & 2,9 & 2,8 & 8,6 & 10,3 & 9,3 \\
\hline \multicolumn{2}{|c|}{ Tamaño muestral } & 37 & 34 & 71 & 93 & 68 & 161 \\
\hline
\end{tabular}

Elaboración propia

\section{Discusión y conclusiones}

El trabajo empírico cuyos resultados aquí se presentan constituye un estudio exploratorio de carácter novedoso, ya que la muestra está compuesta únicamente por estudiantes del Grado en Derecho matriculados en la Universidad de Granada. Es evidente que, a la hora de extraer las conclusiones, hay que ser cautos debido a las limitaciones metodológicas. Así, no cabe duda de que el estudio no puede ser representativo de los estudiantes del Grado en Derecho en general, por lo que, para poder afirmar con rotundidad las dimensiones de percepción de la delincuencia, miedo al delito y actitudes punitivas del estudiantado encuestado, las mismas deberían contrastarse con las respuestas otorgadas por estudiantes de otras universidades. Por 
otra parte, resulta necesario señalar que el muestreo no es probabilístico, dado que, del total de nueve grupos matriculados en el $1^{\text {er }}$ curso del Grado en Derecho, los cuestionarios se repartieron únicamente en tres de esos grupos. Lo mismo cabe decir con respecto al alumnado matriculado en $4^{\circ}$ curso, ya que los cuestionarios se repartieron en el transcurso de distintas asignaturas optativas, en las cuales estaban matriculados alumnos que se encontraban a las puertas de la graduación. De este modo, en el presente trabajo no se ha empleado ningún método estadístico para calcular el tamaño de la muestra, tratándose de un muestreo consecutivo, debido a que se analizan los resultados de varios cursos académicos. Por otra parte, es evidente que puntuales acontecimientos mediáticos relacionados con hechos circunscribibles a la delincuencia grave pueden haber influido en las pautas de respuesta del alumnado.

Con todo, los resultados que arroja la investigación proporcionan una información útil de cara a estudios sucesivos que tengan por objeto analizar las dimensiones de miedo al delito y punitivismo entre determinados sectores de la población. Una hipótesis que podría plantearse es que, también con respecto a los estudiantes encuestados, la imagen que tienen de la delincuencia y de la justicia penal parece provenir en buena medida de la información suministrada por los medios de comunicación, ya que, teniendo en cuenta sus conocimientos jurídicos, los niveles de respuesta en relación con sus preferencias punitivas se acercan a los que arrojarían encuestas semejantes dirigidas a una ciudadanía lega en Derecho. Esto es algo que se observa de forma evidente en los estudiantes de $1^{\text {er }}$ curso. De todos es sabido que la atención dispensada a los hechos delictivos por parte de los mass media no guarda relación directa con la situación de la criminalidad real, sino más bien con determinados acontecimientos o coyunturas sociales y/o políticas, lo cual conduce a que las noticias relacionadas con la criminalidad tengan mayor o menor difusión. En el contexto descrito, los delitos caracterizados por su violencia o dramatismo suelen copar los titulares de prensa en España, dando lugar a presentar a la delincuencia con una magnitud que nada tiene que ver con la situación real (Fuentes Osorio, 2005, p. 3). En consecuencia, no puede considerarse como una hipótesis vacía de contenido el hecho de que la actividad de los medios de comunicación a la hora de presentar la delincuencia en España ha conducido no sólo a un

Revista Española de Investigación Criminológica

Artículo 5, Número 18 (2020)

DOI: https://doi.org/10.46381/reic.v18i0.377

www.criminologia.net

ISSN: 1696-9219 
aumento de la sensación de miedo en la población, sino también a un aumento en sus demandas punitivas. Lógicamente, esta hipótesis podría quedar o no confirmada en sucesivos pases del cuestionario incluyendo en el mismo una pregunta sobre el consumo mediático por parte de los estudiantes de Derecho. Por otra parte, la percepción que los estudiantes tienen de la situación de la delincuencia -en general y violenta- en España no se confirma si se acude a las estadísticas oficiales que informan sobre la evolución de las infracciones delictivas en los últimos años (Cano Paños \& Calvo Alba, 2019, p. 11).

A la hora de confrontar los resultados obtenidos entre el alumnado matriculado en $1^{\text {er }}$ curso con aquellos suministrados por los que se encontraban matriculados en $4^{\circ}$ curso, lo primero que llama la atención es la variación que se produce en las respuestas dirigidas a medir el miedo al delito entre los encuestados. Así, entre $1^{\mathrm{er}}$ y $4^{\mathrm{o}}$ curso se produce una disminución en 19,1 puntos de aquellos que consideran que la situación de la delincuencia en España es «amenazante y peligrosa» (de un 53,8 en el curso 2014/2015 a un 34,7\% en el curso 2017/2018). Como corolario de lo que se acaba de exponer, los partidarios de considerar la situación general de la delincuencia en España como «no peligrosa ni amenazante» pasa de un 19,2\% en el alumnado de $1^{\circ}$ a un $54,1 \%$ en el alumnado de $4^{\circ}$ curso. Resultados similares se obtienen con respecto a la variable dirigida a medir el miedo al delito entre los encuestados, y que hacía referencia al grado de exposición al peligro de un ataque violento por parte de una tercera persona. De este modo, la hipótesis planteada al principio del presente trabajo se vería confirmada con los resultados obtenidos mediante el estudio empírico.

Son varias las razones que podrían explicar esta evolución. A la progresiva adquisición de conocimientos en lo relativo a la delincuencia en España (número de delitos cometidos, incidencia de la delincuencia violenta, etc.) habría que añadir una circunstancia de carácter ambiental: en el caso de los estudiantes matriculados en $1^{\mathrm{er}}$ curso, se está delante de sujetos con edades que no llegan a los 20 años, procedentes de núcleos urbanos con escasa densidad de población, llegado el caso incluso de áreas rurales. De ahí que, en el momento de cumplimentar el cuestionario, estos estudiantes pueden todavía encontrarse sumidos en una especie de shock ambiental debido al cambio que les ha producido en sus vidas el pasar

\section{Revista Española de Investigación Criminológica}

Artículo 5, Número 18 (2020)

DOI: https://doi.org/10.46381/reic.v18i0.377

www.criminologia.net

ISSN: 1696-9219 
de un núcleo de población pequeño a una ciudad como Granada. Por el contrario, en el caso del estudiantado matriculado en el último curso del Grado, y aun procediendo eventualmente de núcleos pequeños de población, estos llevan ya un mínimo de cuatro años viviendo en la ciudad de Granada, por lo que esa dimensión cognitiva del miedo al delito, presente en los alumnos que inician sus estudios, no se produce con respecto a aquellos que se encuentran a las puertas de la graduación.

Uno de los resultados del estudio que cabe en principio calificar como sorprendente hace referencia a las actitudes punitivas de los estudiantes. Así, es digno de destacar el amplio porcentaje del estudiantado de $1^{\text {er }}$ curso que se muestra partidario de la regulación de la PPR en el ordenamiento jurídico español, lo cual da lugar a rebatir aquellos estudios criminológicos que argumentan que la ciudadanía no es punitiva y, por tanto, no demanda penas tan severas como la propia PPR (por todos, Gómez Bellvís \& Falces Delgado, 2019) 9 . Con respecto a este controvertido instituto jurídico hay que señalar que, en su formulación en el cuestionario, se utilizó una pregunta simple y abstracta ${ }^{10}$, renunciando por tanto al empleo de términos que podían influir en los patrones de respuesta del alumnado, o bien a la referencia concreta a casos mediáticos de gran repercusión y con altos niveles de dramatismo. La única explicación plausible a estos altos porcentajes de partidarios de esta pena entre el alumnado de $1^{\text {er }}$ curso habría que buscarla en el hecho de que, aun tratándose de estudiantes del Grado en Derecho, estos carecen todavía de sólidos conocimientos sobre el funcionamiento del sistema penal y los principios que informan la ejecución de las penas privativas de libertad, por lo que dicha actitud punitiva podría estar guiada, en buena medida,

\footnotetext{
${ }^{9}$ Según señalan estos autores en un trabajo reciente, el 60\% de una muestra que debía juzgar la comisión de un delito de asesinato múltiple cometido por parte de una tercera persona, consideraba que la pena máxima y justa para este supuesto era la de 25 o 30 años de prisión, encontrándose la PPR dentro de las opciones de respuesta.

${ }^{10}$ En concreto, la pregunta en torno a la opinión sobre la PPR venía redactada en los siguientes términos: «¿Cuál es su opinión con respecto a la pena de prisión permanente revisable? (1) Debería derogarse del Código Penal; (2) Debería aplicarse obligatoriamente en relación con delitos de especial gravedad, como el asesinato, la delincuencia sexual con víctimas menores de edad o los delitos graves de terrorismo; (3) Considero que, con respecto a determinadas tipologías delictivas de especial gravedad, la pena de prisión permanente revisable constituye incluso una respuesta punitiva no lo suficientemente grave. Debería existir otra pena aún más grave; (4) Todavía no me he formado una opinión».
}

Revista Española de Investigación Criminológica

Artículo 5, Número 18 (2020)

DOI: https://doi.org/10.46381/reic.v18i0.377

www.criminologia.net

ISSN: 1696-9219 
por la información procedente de los medios de comunicación y por la coyuntura política y social del momento.

Ahora bien, uno de los resultados centrales del presente estudio lo proporcionan las respuestas dadas por los estudiantes de $4^{\circ}$ curso en lo relativo a sus niveles de punitivismo. Así, puede observarse cómo el porcentaje de aquellos que se posicionan a favor de derogar la PPR aumenta en 5,1 puntos de $1^{\mathrm{er}}$ a $4^{\mathrm{o}}$ curso, pasando de un $10,7 \%$ a un $15,8 \%$. Más acusadas son las diferencias con respecto a la PM: mientras que entre el estudiantado que comenzó sus estudios de Derecho en el curso 2014/2015 el porcentaje de los partidarios de este controvertido instituto alcanzó un 39,8\%, dicho porcentaje baja de forma acusada cuatro años más tarde, para situarse en un 26,5\% en el curso 2017/2018. De este modo, la hipótesis planteada al comienzo del presente trabajo se vería de nuevo confirmada con los datos expuestos.

Una de las posibles explicaciones a esta disminución en las actitudes punitivas de los estudiantes de Derecho podría venir de su progresiva formación académica en temáticas jurídicas de primer orden como son los derechos fundamentales de la persona, los fines de la pena o bien cuestiones de carácter procesal-penal y penitenciario. Con todo, no deja de sorprender que, entre el alumnado que se encuentra a las puertas de la graduación, sigan siendo más de un 60\% los partidarios de aplicar la PPR, tal y como la misma está actualmente regulada en el Código Penal.

Por otro lado, las diferencias entre sexos, las cuales, en el caso del estudiantado matriculado en $1^{\text {er }}$ curso, evidenciaban una actitud ligeramente más punitiva de las mujeres con respecto a la PPR y a la aprobación de la pena capital como condena estipulada en el Código Penal, se mantienen con respecto al alumnado de sexo femenino matriculado en cursos superiores. Y es que, como cabía esperar, el estudiantado de sexo femenino presenta unos niveles de miedo al delito superiores al de sexo masculino, lo cual a buen seguro repercute en sus actitudes punitivas. De este modo, los resultados alcanzados en el presente trabajo vendrían a contradecir otros estudios que señalan que las mujeres, por regla general, son menos firmes frente al delito que los hombres (Serrano Maíllo, 2016, pp. 438-439).

Revista Española de Investigación Criminológica

Artículo 5, Número 18 (2020)

DOI: https://doi.org/10.46381/reic.v18i0.377

www.criminologia.net

ISSN: 1696-9219 
El mayor miedo al delito en mujeres que en hombres ha podido ratificarse a través de los datos que señalan que la percepción que las mujeres tienen sobre la situación en general de la delincuencia en España difiere con respecto a los varones, en el sentido de que estos últimos consideran en mayor medida que dicha situación no es ni amenazante ni peligrosa. Por otro lado, las mujeres perciben en mucha mayor medida que los hombres un alto riesgo de ser víctimas de un ataque violento por parte de una tercera persona. Tal y como han señalado ya otros estudios, la especificidad de género con respecto a la variable referida al miedo al delito podría ser causada por la mayor vulnerabilidad de las mujeres, pero también por efectos de la socialización en el sentido de la transmisión social y cultural de un rol específico por exclusiva razón de género (Streng, 2014, p. 70).

Por último, debe tenerse en cuenta que la última fase de los resultados cuenta con dos limitaciones relacionadas con el tamaño y la composición de la muestra: (1) Dado el reducido tamaño de algunas de las submuestras obtenidas, los análisis serán contrastados con la ampliación de la muestra en la siguiente edición para evitar errores de tipo I; (2) No debe olvidarse que en los cursos encuestados del Grado en Derecho existe una sobrerrepresentación de mujeres en la muestra, por lo que las conclusiones adoptadas en este aspecto deben ser tomadas con cierta cautela hasta que se pueda confirmar la tendencia con la próxima ampliación de la muestra.

\section{Revista Española de Investigación Criminológica}




\section{Referencias}

Aizpurúa, E. (2015) "Delimitando el punitivismo. Las actitudes de los españoles hacia el castigo de los infractores juveniles y adultos", Revista Española de Investigación Criminológica, núm. 13, págs. 1-30.

Aizpurúa González, E. \& Fernández Molina, E. (2011) "Información, ¿antídoto frente el "populismo punitivo"? Estudio sobre las actitudes hacia el castigo de los menores infractores y el sistema de Justicia Juvenil", Revista Española de Investigación Criminológica, núm. 9, págs. 1-29.

Applegate, B., Cullen, F. \& Fisher, B. (2002) "Public views toward crime and correctional policies. Is there a gender gap?”, Journal of Criminal Justice, núm. 30, págs. 89-100.

Barberet, R. Fisher, B. \& Taylor, H. (2004) University student safety in the East Midlands, Home Office Online Report 61/04.

Cano Paños, M. A. \& Calvo Alba, M. A. (2019) "Percepción de la delincuencia, miedo al delito y actitudes punitivas en España. Resultados de una encuesta realizada a estudiantes del Grado en Derecho entre los años 2015 y 2018”, InDret, núm. 4, págs. $1-42$.

Cohen, J. (1988): Statistical Power Analysis for the Behavioral Sciences, $2^{\mathrm{a}}$ Ed., New Jersey: Lawrence Erlbaum Associates.

Cullen, F., Cullen, J. \& Wozniak, J. (1988) "Is rehabilitation dead?: The myth of punitive public", Journal of Criminal Justice, Vol. 16, págs. 303-317.

Chan-Gamboa, E., Morales-Quintero, L., Ruiz-Pérez, J. \& Vaca-Cortés, J. (2017) “Factores sociodemográficos asociados a la victimización delictiva en estudiantes universitarios de tres ciudades mexicanas", Pensamiento Psicológico, Vol 15, No 2, 2017, págs. 93107.

Chen, G. \& Einat, T. (2015) "The relationship between criminology studies and punitive attitudes”, European Journal of Criminology, Vol. 12(2) págs. 169-187

Díez Ripollés, J. L., Girón González-Torre, F., Stangeland, P. \& Cerezo Domínguez, A. (1996) Delincuencia y víctimas (Encuestas de victimización en Málaga), Valencia, Tirant lo Blanch.

Fernández Molina, E. \& Tarancón Gómez, P. (2010) "Populismo punitivo y delincuencia juvenil: mito o realidad”, Revista Electrónica de Ciencia Penal y Criminología, núm. 12, págs. 1-25.

Fuentes Osorio, J. (2005) "Los medios de comunicación y el Derecho penal”, Revista Electrónica de Ciencia Penal y Criminología, núm. 7, págs. 1-51.

García España, E. (2013) “¿Los conocimientos criminológicos modulan la actitud punitiva?”, Estudios sobre el mensaje periodístico, Vol. 19, Núm. Especial abril, Madrid, Servicio de Publicaciones de la Universidad Complutense, págs. 753-761.

Revista Española de Investigación Criminológica

Artículo 5, Número 18 (2020)

DOI: https://doi.org/10.46381/reic.v18i0.377

www.criminologia.net

ISSN: 1696-9219 
García España, E., Pérez Jiménez, F. \& Benítez Jiménez, M. J. (2006) La delincuencia según las víctimas: Un enfoque integrado a partir de una encuesta de victimización. Informe $O D A$ 2006, Málaga, Instituto Andaluz Interuniversitario de Criminología, Fundación El Monte.

Gómez Bellvís, A. \& Falces Delgado, C. (2019) "Los efectos del contexto en la expresión de las actitudes punitivas: El caso del apoyo ciudadano a la prisión permanente revisable", Revista Electrónica de Criminología, 01-01, págs. 1-14.

Igareda, N. \& Bodelón, E. (2014) "Las violencias sexuales en las universidades: cuando lo que no se denuncia no existe", Revista Española de Investigación Criminológica, núm. 12, págs. 1-27.

Kohm, S., Waid-Lindberg, C., Weinrath, M., O’Connor, S., \& Dobbs, R. (2012) “The Impact of Media on Fear of Crime among University Students: A Cross-National Comparison", Canadian Journal of Criminology and Criminal Justice, Vol. 54, Núm. 1, págs. 67-100.

Kury, H., Obergfell-Fuchs, J., \& Smartt, U. (2002) "The evolution of public attitudes to punishment in Western and Eastern Europe", en: Roberts, Julian\&Hough, Mike (eds.), Changing attitudes to punishment. Public opinion, crime and justice, Cullompton, Devon: Willan, págs. 93-114.

Luque Reina, E. (1999) Les víctimes del delicte, Col.lecció Justícia i Societat, núm. 20, Generalitat de Catalunya, Centre d'Estudis Jurídics i Formació Especialitzada.

Roberts, J. \& Hough, M. (2005) Understanding public attitudes to criminal justice, New York/Maidenhead, Open University Press.

Serrano Maíllo, A. (2016) Firmeza frente al delito y comunidad en la modernidad reflexiva. La tesis extendida de los sentimientos de inseguridad como teoría del control social, Madrid: Dykinson.

Streng, F. (2014) Kriminalitätswahrnehmung und Punitivität im Wandel. Kriminalitäts- und berufsbezogene Einstellungen junger Juristen», Heidelberg, Kriminalistik.

Varona Gómez, D. (2008) "Ciudadanos y actitudes punitivas: Un estudio piloto de población universitaria española”, Revista Española de Investigación Criminológica, núm. 6, págs. 1-38.

Varona Gómez, D. (2009) “Somos los españoles punitivos? Actitudes punitivas y reforma penal en España”, InDret, núm. 1, págs. 1-31.

Varona Gómez, D. (2013) "Percepción y elección del castigo en España: Resultados a partir de la Encuesta Social Europea (5 $5^{\text {a }}$ E.)”, Cuadernos de Política Criminal, núm. 111, págs. 145-193.

Varona Gómez, D. (2016) El debate ciudadano sobre la justicia penal y el castigo: razón y emoción en el camino hacia un Derecho penal democrático, Madrid, Marcial Pons.

Revista Española de Investigación Criminológica

Artículo 5, Número 18 (2020)

DOI: https://doi.org/10.46381/reic.v18i0.377

www.criminologia.net

ISSN: 1696-9219 
Villacampa, C. \& Pujols, A. (2017) "Prevalencia y dinámica de la victimización por stalking en población universitaria", Revista Española de Investigación Criminológica, núm. 15, págs. 1-27.

\section{Agradecimientos}

Este trabajo ha sido posible gracias a la colaboración de los compañeros y compañeras de la Facultad de Derecho de la Universidad de Granada, los/las cuales permitieron llevar a cabo el estudio en las franjas horarias en las que impartieron docencia en los distintos grupos aquí encuestados.

\section{Financiamiento}

Este trabajo se enmarca dentro del Proyecto «Criminología, evidencias empíricas y Política criminal. Sobre la incorporación de datos científicos para la toma de decisiones en relación con la criminalización de conductas». Referencia: DER2017-86204-R, financiado por la Agencia Estatal de Investigación (AEI)/Ministerio de Ciencia, Innovación y Universidades y la Unión Europea a través del Fondo Europeo de Desarrollo Regional - FEDER - «Una manera de hacer Europa». Investigador principal: Fernando Miró Llinares.

Miguel Ángel Cano Paños es Doctor en Derecho por la Universidad de Potsdam (Alemania) y Doctor en Criminología por la Universidad de Murcia. Desde el año 2014 es Profesor Titular de Derecho Penal y Criminología en la Universidad de Granada. Sus ámbitos de investigación son la delincuencia terrorista y el crimen organizado, la delincuencia y el derecho penal de menores, así como, desde un ámbito estrictamente criminológico, el punitivismo, el miedo al delito, así como la calidad de vida penitenciaria. Los resultados de estas investigaciones han visto la luz en más de 100 contribuciones científicas, publicadas tanto en España como en el extranjero.

iD

https://orcid.org/0000-0001-5426-7227

Ma Ángeles Calvo Alba es Graduada en Sociología por la Universidad de Granada y Máster en Problemas Sociales por la misma Universidad. Desde el año 2015, Calvo Alba colabora activamente como socióloga analista de datos en el Instituto Andaluz Interuniversitario de Criminología, Sección Universidad de Granada. Además, es Manager Research y cofundadora de la firma Equality Research, con la que ha desarrollado el Proyecto "La incidencia de la violencia de género en colectivos vulnerables: mujeres mayores y con diversidad funcional/discapacitadas" (2020), para el Centro Asesor de la Mujer en Ceuta.

iD

https://orcid.org/0000-0002-0439-2297

\section{Revista Española de Investigación Criminológica}

Artículo 5, Número 18 (2020)

DOI: https://doi.org/10.46381/reic.v18i0.377

www.criminologia.net

ISSN: 1696-9219 